\title{
Mindset Kinetics and Crime Behavior-Quantitative Methods? A New Forensic Quantitative Approach. How Biochemistry, Toxicology, Imaging Principle Can Help in Jurisdictional Settings
}

\author{
Luisetto $M^{* 1}$, Naseer Almukhtar ${ }^{2}$ and Farhan Ahmad Khan ${ }^{3}$ \\ ${ }^{1}$ Applied Pharmacologist, Independent Researcher, Italy \\ ${ }^{2}$ Professor, Physiology, College of Medicine, University of Babylon, Iraq \\ ${ }^{3}$ Department of Pharmacology, Government Medical College, India \\ *Corresponding author: Mauro Luisetto, Applied Pharmacologist, Independent Researcher, Italy
}

ARTICLE INFO

Received: 㗀 March 09, 2019

Published: 慧 March 19, 2019

Citation: Luisetto M, Naseer A, Farhan Ahmad K. Mindset Kinetics and Crime Behavior-Quantitative Methods? A New Forensic Quantitative Approach. How Biochemistry, Toxicology, Imaging Principle Can Help in Jurisdictional Settings. Biomed J Sci \& Tech Res 16(2)2019. BJSTR. MS.ID.002810.

\section{ABSTRACT}

In some non-voluntary crime is crucial verify the amygdala and limbic system physiopathology of human responsible of the facts under Jurisdictional contest. But is relevant to verify this condition in objective way. Many human physio-pathological conditions are deeply investigated using biochemical or imaging technique is possible that today many organ and system are currently evaluated with high number of biomedical, instrumental or imaging test also In basal or stressing condition. The objective setting of amygdala and limbic system must be added in jurisdictional procedure not as a prove but an additional information to be correctly evaluated.

Keywords: Crime; Mindset Kinetics; Quantitative Methods; Physio Pathology; Jurisdictional

\section{Introduction}

Often is possible to say: One second after often is too much In many crime situation mindset kinetics of amygdala, limbic system, thalamus is a crucial aspect to be adequately considered in jurisdictional settings. This neuronal system is involved in manage of anger, impulsivity, aggressiveness, fear and other emotional response to various kind of stimulous. In article "Amygdala Hijack and the Fight or Flight Response" By Arlin Cuncic 2018: "The term "amygdala hijack" was introduced by the psychologist D. Goleman .He used the term to recognize that although we have evolved as humans, we retain an ancient structure in our brain that is designed to respond swiftly to a threat. While at one time this was designed to protect us, it can interfere with our functioning in the modern world where threats are often more subtle in nature. The amygdala is involved in the brain fear circuit, responsible for the fight-or-flight response that causes to respond to threats. The amygdala is responsible for deciding what memories are stored and where they are stored. The level of emotion that is attached to a memory determines where it is stored in the CNS. When faced with a threatening situation, thalamus, which receives incoming stimuli, sends signals to the amygdala and the cortex. If amygdala senses danger, it makes a split second decision and begins the fightor-flight response before the cortex has time to over-rule it. This cascade of events triggers the release of adrenaline, which leads to increased heart rate, blood pressure, and breathing. racing heart, shaking, sweating, and nausea as this happens. The amygdala triggers a sudden and intense unconscious emotional response that shuts off the cortex, making it hard for you to think clearly about the situation. The brain triggers the release of stress hormones like cortisol, you find it increasingly hard to problem solve and concentrate. 
This whole process takes a toll and is possible not recover to original level of functioning for several hours. Chronic stress also plays a role in the functioning of fear circuitry in the brain. People with post-traumatic stress disorder (PTSD) show greater amygdala activation and therefore, increased emotional responding including fear and anxiety responses. Those with other anxiety disorders, such as social anxiety disorder (SAD) and panic disorder (PD) may also respond more strongly in their amygdala. Chronic stress can lead to an over-active fear and anxiety circuit in your brain, which also reduces the functioning of other areas of the brain that help with inhibition of fear, such as the hippocampus and medial prefrontal cortex. Chronic stress can trigger more frequent amygdala hijacks and even subsequent problems with short-term memory." Is possible to say that this fact since today is not measured or quantized in jurisditional contest? To differ pathological from physiological individuals? In how many situations if available more time to think different actions could result?

To manage different and many informations in few seconds, or to control anger or other emotional reaction can be difficult for various subjects. (rapid circuits thalamus -amygdala and slow circuits involving also frontal cortex). Sensorial information arrives from thalamus to amygdala and from this start motor signals. Amygdala compare also past experience with the new intense stimulous. Related this consideration is very important to ask to justice administration to introduce objective methods to verify individual's reactivity in high stressing condition. A real measure of this condition makes possible to differ individual with normal management of minkset kinetics also in high stressant condition from whom present an abnormal reaction.

\section{Material and Methods}

With an observational approach some relevant literature on PUBMED is analized to verify relationship between some crime behavior and amigdala and limbic system activation. After this review is submitted to researcher a global conclusion related to the topic of this work.

\section{Results}

From literature: In article Amygdala pharmacology and crime behavior, dysfunctions to be considered as a disease.? Is reported that: "we conclude that is needed to find an objective diagnostic system to verify the basic level of activation status of amygdala in stress conditions and also to find if a drugs therapy system can be considered if we have an organic pathology condition. As others physiological apparatus: a pathological activation and status can be controlled by specific pharmacological therapy" [1] and related mindset kinetics article "Scientist like M. Planck, Einstein, Bohr, De Broglie, Schrödinger, Heisenberg et others (involved in theory of quantum chemical physic field ), E. goldratt (Teory of constraints), Michaelis-Menten ( a biochemistry kinetics theory), other biochemical and enzymatic reaction theory must be deeply investigated also in other fields like neuroscience and applied in order to better clear some process. The factor that can join the organic theories to the psychological approach can be an abnormal- pathological mindset kinetics process or an overuse or saturation in some Psyco- neurological process. And The single resilience ability (or single buffer properties) in biochemical receptorial status must be also objectivable So a better objective way to verify the amount of stressing condition, time of exposure and quality must be introduced. (in differente field like Psycology, psychiatry, forensic).

A useful instrument for healthcare professionals and patients. (using also a toxicological approach: what toxic condition, amount and time of exposure and under a biochemical aspect (kinetics, max velocity of a system, saturation) and not only the receptorial status. Observing biochemistry kinetics law can we think that a Zero Order Kinetics in mindset kinetics can reduce some mind-brain disorder? (Only a determinate quantum of stressing condition in a definite amount of time: really short, medium or prolonged)" [2]. According Uri Maoz "A defendant is criminally responsible for his action only if he is shown to have engaged in a guilty act-actus reus (eg for larceny, voluntarily taking someone else's property without permission)-while possessing a guilty mind-mens rea (eg knowing that he had taken someone else's property without permission, intending not to return it)-and lacking affirmative defenses ( in eg the insanity defense or self-defense). We first review neuroscientific studies that bear on the nature of voluntary action, and so could, potentially, tell us something of importance about the actus reus of crimes. Then we look at studies of intention, perception of risk, other mental states that matter to the mens rea of crimes. we discuss studies of self-control, which might be relevant to some formulations of the insanity defense. As we show, to date, very little is known about the brain that is of significance for understanding criminal responsibility.

But there is no reason to think that neuroscience cannot provide evidence that will challenge our understanding of criminal responsibility" [3]. Sergio Canavero written : "Although the definition of criminal behavior is fraught with controversy, with single acts "criminalized" or "decriminalized" according to time and place, and as such being observed in individuals of all sorts, there seems to be an agreement across the board that the truly dangerous subjects are psycho-paths and the subjects affected by the Antisocial Personality Disorder"[4] And Rebecca Roache showed that: "L Berlin reports that neuro-scientific data play an increasing role in court. They have been used to argue that criminals are not morally responsible for their behaviour because their brains are 'faulty', and there is evidence that such data lead judges to pass more lenient sentences. I raise 2 concerns about the view that neuroscience can show criminals not to be morally responsible. That the brains of (say) violent criminals differ from most people's brains does not straightforwardly show that violent criminals are less morally responsible. Behavioral states arise inter alia from brain states, and since violent criminals' behavioral states differ from those of most people, it is unsurprising that violent criminals' brains should differ 
from most people's brains. This no more shows violent criminals to have diminished moral responsibility than differences between the brains of cheerful and uncheerful people show either group to have diminished moral responsibility. Those who view brain abnormalities as evidence of reduced moral responsibility rely on the assumptions that people with normal brains have free will and that we know what sorts of brain activity undermine free will. both of these assumptions are highly controversial.

As a result, neuroscience is not a reliable source of information about moral responsibility. until we settle whether and under what circumstances brain activity is incompatible with free will, neuroscience cannot tell us anything useful about criminal accountability." [5] according Dean Mobbs "Archaeological discoveries of traumatic injuries in primitive hominid skulls strongly hint that our species has a long history of violence. Despite repeated attempts throughout history, including efforts to eliminate violence through the imposition of criminal sanctions, we have yet to dispel our violent nature. Consequently, criminal violence remains a common feature of most societies. As policy-makers seek deeper understandings of criminally violent and anti-social behavior, many contemporary neuro-scientists assume that the essential ingredients of the human condition, including free will, empathy, and morality, are the calculable consequences of an immense assembly of neurons firing. Intuitively, this view opposes Cartesian dualism (the brain and mind are separate, but interacting, entities) and assumes that violence and antisocial behavior emanate from a mechanistically determined brain" [6]. Robert K. Naumann et al showed that: A Primer on the reptile brain, in particular the light it sheds on the structural and functional evolution of the vertebrate neural circuits.

"Deep inside the skull of every one of us there is something like a brain of a crocodile. Surrounding the R-complex is the limbic system or mammalian brain, which evolved tens of millions of years ago in ancestors who were mammal but not yet primates. It is a major source of our moods and emotions, of our concern and care for the young. And finally, on the outside, living in uneasy truce with the more primitive brains beneath, is the cerebral cortex; civilization is a product of the cerebral cortex. "C. Sagan's amusing words of wisdom notwithstanding is the $\mathrm{H}$-bomb not also a product of the cerebral cortex? is the reptilian brain really just a mammalian brain missing most of the parts? Some 320 million years ago, the evolution of a protective membrane surrounding the embryo, the amnion, enabled vertebrates to develop outside of water and thus to invade new terrestrial niches. These amniotes were the ancestors of today's mammals and sauropsids (reptiles and birds). Presentday reptiles are a diverse group of more than 10,000 species that comprise sphenodons ('Tuatara'), lizards, snakes, turtles and crocodilians.

Although turtles were once thought to be the most 'primitive among reptiles, current genomic data point toward two major groupings: the Squamata (lizards and snakes); and a group comprising both the turtles and the Archosauria (dinosaurs, modern birds and crocodilians). Dinosaurs inhabited the Earth from the Triassic (230 million years ago), at a time when the entire landmass formed a single Pangaea. They flourished from the beginning of the Jurassic to the mass extinction at the end of Cretaceous (65 million years ago), and birds are their only survivors" [7] According Hoerst M: "Emotional dysfunction in a fronto-limbic network has been implicated in the pathophysiology of borderline personality disorder (BPD). The amygdala is a key region of the limbic system and plays an important role in impulsivity, affect regulation, and emotional information processing and thus is likely related to BPD symptoms. Alterations of the metabolism in the amygdala might be of interest for understanding the pathophysiology of BPD. the amygdala is a difficult region from which to acquire magnetic resonance spectra. We implemented a method for proton magnetic resonance spectroscopy ((1)H MRS) at 3.0 T in which we acquire data within only the small amygdala. The purpose of this study was to determine alterations of the metabolism in the amygdala in BPD patients.

Twenty-one unmedicated BPD patients and 20 age-matched healthy control participants underwent (1)H MRS to determine neurometabolite concentrations in the left amygdala. All participants underwent psycho-metric assessments. Significantly reduced total $\mathrm{N}$-acetylaspartate ( $\mathrm{tNAA}$ ) and total creatine $(\mathrm{tCr})$ concentrations in the left amygdala of patients with BPD were founded. BPD patients with comorbid post-traumatic stress disorder (PTSD) showed lower levels of $\mathrm{tCr}$ compared with BPD patients without PTSD and healthy control subjects. No significant correlations between neurochemical concentrations and psychometric measures were founded. Decreased tNAA and tCr might indicate disturbed affect regulation and emotional information processing in the amygdala of BPD patients. These findings are consistent with many functional and structural neuro-imaging studies and may help to explain the greater emotional reactivity of BPD patients" [8-11]. Sakai $\mathrm{Y}$ "The present work was performed to assess cerebral glucose metabolism in patients with panic disorder using positron emission tomography. F-fluoro-deoxyglucose positron emission tomography with voxel-based analysis was used to compare regional brain glucose utilization in 12 nonmedicated panic disorder patients, without their experiencing panic attacks during positron emission tomography acquisition, with that in 22 healthy controls.

Panic disorder patients showed appreciably high state anxiety before scanning and exhibited significantly higher levels of glucose uptake in the bilateral amygdala, hippocampus, thalamus, in midbrain, caudal pons, medulla, and cerebellum than controls. These results provided the first functional neuroimaging support in human patients for the neuroanatomical hypothesis of panic disorder focusing on the amygdala-based fear network" [12]. And Sofi da Cunha-Bang. The ability to successfully suppress impulses and angry affect is fundamental to control aggressive reactions following provocations. The aim of this work was to examine neural 
responses to provocations and aggression using a laboratory model of reactive aggression. We used a novel functional magnetic resonance imaging point-subtraction aggression paradigm in 44 men, of whom 18 were incarcerated violent offenders and 26 were control non-offenders. We measured brain activation following provocations (monetary subtractions), while the subjects had the possibility to behave aggressively or pursue monetary rewards. The violent offenders behaved more aggressively than controls (aggression frequency 150 vs $84, \mathrm{P}=0.03$ ) and showed significantly higher brain reactivity to provocations within the amygdala and striatum, as well as reduced amygdala-prefrontal and striatoprefrontal connectivity. Amygdala reactivity to provocations was positively correlated with task-related behavior in the violent offenders. Across groups, striatal and the prefrontal reactivity to provocations was positively associated with trait anger and trait aggression. These results suggest that violent individuals display abnormally high neural sensitivity to social provocations, a sensitivity related to aggressive behavior.

These findings provide new insight into the neural pathways that are sensitive to provocations, which is critical to more effectively shaped interventions that aim to reduce pathological aggressive behavior "[13]. Larry J Siever showed that "Acts of violence account for an estimated 1.43 million deaths world-wide annually. While violence can occur in many contexts, individual acts of aggression account for the majority of instances. In some individuals, repetitive acts of aggression are grounded in an underlying neurobiological susceptibility that is just beginning to be understood. The failure of "top-down" control systems in the prefrontal cortex to modulate aggressive acts that are triggered by anger provoking stimuli appears to play an important role. An imbalance between prefrontal regulatory influences and hyper-responsivity of the amygdala and other limbic regions involved in affective evaluation are implicated. Insufficient serotonergic facilitation of "top-down" control, excessive catecholaminergic stimulation, and subcortical imbalances of glutamatergic/ gabaminergic systems as well as pathology in neuropeptide systems involved in the regulation of affiliative behavior may contribute to abnormalities in this circuitry.

Pharmacological interventions such as mood stabilizers, which dampen limbic irritability, or selective serotonin reuptake inhibitors (SSRIs), which may enhance "top-down" control, as well as psychosocial interventions to develop alternative coping skills and reinforce reflective delays may be therapeutic. Structural imaging-While lesion studies evaluate effects of damage to specific brain regions, structural imaging paradigms measure naturalistic variability in volumes and shapes of brain structures. Reductions in prefrontal gray matter that are presumably based in aberrant development have been reported in individuals with antisocial personality disorder and are often associated with autonomic deficits. Significant volume reductions have been demonstrated in the left orbital frontal- cortex and right anterior- cingulate cortex in patients with borderline personality disorder, most marked in
Brodmann's area 24, which has also been implicated in functional imaging studies of these patients. The temporal cortex, particularly in the medial temporal cortex and hippocampus, has also been demonstrated to have structural alterations, including altered asymmetry in antisocial subjects. Orbital frontal/cingulate cortical processing efficiency-Functional brain imagining permits an assessment of brain activation patterns in specific regions of interest in individuals with a history of recurrent episodic violent behavior.

Initial studies focusing on psychiatric patients with a history of violence demonstrated decreased glucose metabolism using a positron emission tomography with [14-18] fluorodeoxyglucose (FDG-PET) paradigm in the temporal and frontal cortices. Similar results were obtained in a study of murderers. An inverse relationship has also been reported between the history of impulsive aggressive behavior and glucose metabolism in the orbital frontal cortex and right temporal cortex, with reductions of metabolism in prefrontal Brodmann's areas 46 and 6 in patients with borderline personality disorder. In a PET study evaluating responses to the probe metachlorophenylpiperazine, decrements in the lateral, medial, and orbital frontal cortices were found at baseline in men with a history of physical aggression and in the orbital frontal cortex for both men and women with a history of physical aggression. In an imaging study of an unrestrained aggressive scenario, healthy volunteers demonstrated blood flow reductions in the orbital frontal cortex, suggesting a release of "top-down" control of aggression. While participating in a laboratory designed to provoke aggression, the Point Subtraction Aggression Paradigm, patients with intermittent explosive disorder, as determined by integrated research criteria, and borderline personality disorder made more aggressive responses relative to comparison subjects. In the Point Subtraction Aggression Paradigm, patients with borderline personality disorder who were characterized by anger dys-control showed diminished responses to provocation in the medial frontal cortex and the anterior frontal cortex relative to comparison subjects but greater responses in the orbital frontal cortex, presumably to dampen aggressive responses.

In an fMRI paradigm, when viewing negative pictures compared with rest, patients with borderline personality disorder showed greater activity relative to healthy comparison subjects in the amygdala, fusiform gyrus, para-hippocampul gyrus, cerebellar declive, ventro-lateral prefrontal cortex, occipital visual areas, and regions related to sensory, emotional, and facial processing, while healthy comparison subjects showed greater activity in the insula, involved in visceral emotional processing, and the dorso-medial/ dorsolateral pre-frontal cortex, involved in cognitive processing" [19]. "Recent evidence suggests that putting feelings into words activates the prefrontal cortex (PFC), and suppresses the response of the amygdala, potentially helping to alleviate emotional distress. To further elucidate the relationship between brain structure and function in these regions, structural and functional magnetic resonance imaging (MRI) data were collected from a sample of 
20 healthy human subjects. Structural MRI data were processed using cortical pattern matching algorithms to produce spatially normalized maps of cortical thickness. During functional scanning, subjects cognitively assessed an emotional target face by choosing one of two linguistic labels (label emotion condition) or matched geometric forms (control condition). Manually prescribed regions of interest for the left amygdala were used to extract percent signal change in this region occurring during the contrast of "label emotion" versus "match forms."

A correlation analysis between left amygdala activation and cortical thickness was then performed along each point of the cortical surface, resulting in a color-coded $r$ value at each cortical point. Correlation analyses revealed that gray matter thickness in left ventro-medial PFC was inversely correlated with task-related activation in the amygdala. These data add support to a general role of the ventro-medial PFC in regulating activity of the amygdala" [20]. Roee Admon: "An intact amygdala is thought to be critical for advantaged processing of relevant stimuli in the environment via efficient tagging of its emotional nature, whereas the hippocampus was found to be involved in forming episodic representations of emotional events by tagging contextual meaning to it . Therefore, it is possible that during the second time point (i.e., After Stress), the limbic response to a context in which the stressful experience took place (i.e., medical) is related to the fact that this context is a reminder of the stressful event, thus evoking emotions. This is further supported by our finding that the individual amygdala and hippocampus activation levels After Stress were positively correlated with the change in stress symptoms over time, implying that the more emotionally significant the context is for the individual, the more intensely those regions respond to reminders of it. Similarly, the levels of amygdala activation and hippocampal volume were found to be correlated with symptom severity in PTSD. Our current findings, however, show that even in the healthy brain the limbic response to stress-related content within a few months after the occurrence of a stressful life event is related to an increase in stress symptoms. In that regard it is important to note, however, that our participants were all adolescents, and it is widely recognized that during adolescence the brain shows remarkable changes in both structure and function.

Thus, stressors experienced during adolescence may have a different neural impact than when experienced at adulthood, and future studies should examine the age generality of our findings in older cohorts" [21]. "This study sought to investigate neural activity in the amygdala during episodes of mania. Nine manic subjects and nine healthy comparison subjects underwent functional magnetic resonance imaging (fMRI) while performing a neuropsychological paradigm known to activate the amygdala. Subjects viewed faces displaying affect (experimental task) and geometric forms (control task) and matched them to one of two simultaneously presented similar images. Manic subjects had significantly increased activation in the left amygdala and reduced bilateral activation in the lateral orbitofrontal cortex relative to the comparison subjects. Increased activation in the amygdala and decreased activation in the orbitofrontal cortex may represent disruption of a specific neuroanatomic circuit involved in mania. These brain regions may be implicated in disorders involving regulation of affect" [22]. "The ability to successfully suppress impulses and angry affect is fundamental to control aggressive reactions following provocations.

The aim of this study was to examine neural responses to provocations and aggression using a laboratory model of reactive aggression. We used a novel functional magnetic resonance imaging point-subtraction aggression paradigm in 44 men, of whom 18 were incarcerated violent offenders and 26 were control nonoffenders. We measured brain activation following provocations (monetary subtractions), while the subjects had the possibility to behave aggressively or pursue monetary rewards. The violent offenders behaved more aggressively than controls (aggression frequency 150 vs $84, \mathrm{P}=0.03$ ) and showed significantly higher brain reactivity to provocations within the amygdala and striatum, as well as reduced amygdala-prefrontal and striato-prefrontal connectivity. Amygdala reactivity to provocations was positively correlated with task-related behavior in the violent offenders. Across groups, striatal and prefrontal reactivity to provocations was positively associated with trait anger and trait aggression. These results suggest that violent individuals display abnormally high neural sensitivity to social provocations, a sensitivity related to aggressive behavior.

These findings provide novel insight into the neural pathways that are sensitive to provocations, which is critical to more effectively shaped interventions that aim to reduce pathological aggressive behavior "[23]. M Brower: "To establish the link between frontal lobe dysfunction and violent and criminal behaviour, based on a review of relevant literature. Articles relating evidence of frontal lobe dysfunction with violence or crime were collected through a MEDLINE search using the keyword "frontal lobe" combined with the terms "aggression," "violence," "crime," "antisocial personality disorder," "psychopathy," "impulse control disorders", and "episodic dyscontrol." Reference lists were then searched for additional articles. High rates of neuropsychiatric abnormalities reported in persons with violent and criminal behaviour suggest an association between aggressive dyscontrol and brain injury, especially involving the frontal lobes. The studies reviewed support an association between frontal lobe dysfunction and increased aggressive and antisocial behaviour. Focal orbitofrontal injury is specifically associated with increased aggression. Deficits in frontal executive function may increase the likelihood of future aggression, but no study has reliably demonstrated a characteristic pattern of frontal network dysfunction predictive of violent crime.

Clinically significant focal frontal lobe dysfunction is associated with aggressive dyscontrol, but the increased risk of violence seems less than is widely presumed. Evidence is strongest for an 
association between focal prefrontal damage and an impulsive subtype of aggressive behaviour" [24] (Figure 1). Hirono N "Aggressive behavior is common in patients with dementia disease. Temporo-limbic and prefrontal cortical lesions can produce pathological aggression; involvement of these structures has not been established in aggressive patients with dementia. To study the relation between the regional brain perfusion and aggressive behavior in patients with dementia. We compared the pattern of regional cerebral perfusion determined with technetium Tc 99m-labeled hexamethylpropelene amineoxime single photon emission computed tomography in 2 groups of 10 patients with dementia with and without aggression, that were comparable for demographic factors, severity of cognitive impairments, and other behavioral symptoms as measured by the Neuro-psychiatric Inventory. Patients with aggression revealed significant $(\mathrm{P}<.001)$ hypo-perfusion in the left anterior temporal cortex; additional bilateral dorso-frontal and right parietal cortex were also found to be significantly hypo-perfused. These results indicated an association between aggression and decreased perfusion in the left anterior temporal cortex "[25].
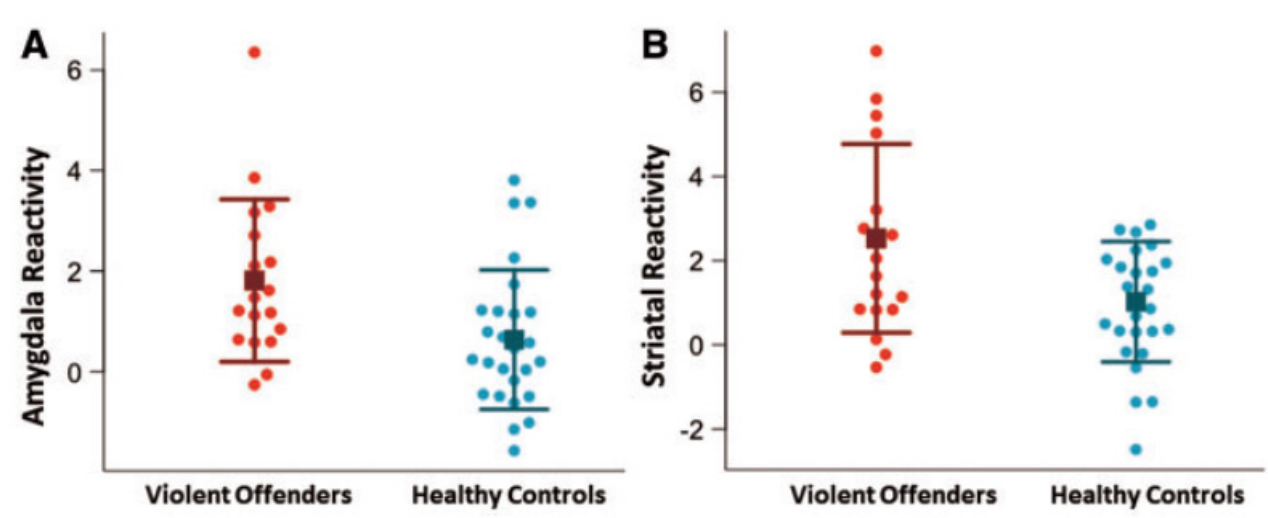

A. "Amygdala reactivity" represents signal values extracted from left and right amygdala clusters significantly activated in response to provocations across all participants. Violent offenders show significantly higher amygdala reactivity to provocations ( $\mathrm{P} 1 / 40.02)$.

B. "Striatal Reactivity" represents signal values extracted from left and right striatal clusters significantly activated in response to provocations across all participants. Violent offenders show significantly higher amygdala reactivity to provocations (P1/40.02). Squares represent group mean and error bars represent standard deviations [18].

Figure 1: Heightened amygdala and striatal reactivity to provocations in violent offenders.

Major damage to gray and white matter in the pre-frontal cortex and autonomic deficits has been found to result in pseudopsychopathic personality in patients with neurological disorders, but it is not known whether people with antisocial personality disorder (APD) in the community who do not have discernable brain trauma also have subtle prefrontal deficits. Prefrontal gray and white matter volumes were assessed using structural magnetic resonance imaging in 21 community volunteers with APD and in 2 control groups, comprising 34 healthy subjects, 26 subjects with substance dependence (substance-dependent group), and 21 psychiatric controls. Autonomic activity (skin conductance and heart rate) was also assessed during a social stressor in which participants gave a video-taped speech on their faults. The APD group showed an $11.0 \%$ reduction in prefrontal gray matter volume in the absence of ostensible brain lesions and reduced autonomic activity during the stressor. These deficits predicted group membership independent of psycho-social risk factors. these findings provide the first evidence for a structural brain deficit in APD. This prefrontal structural deficit may underlie the low arousal, poor fear conditioning, lack of conscience, and decision- making deficits that have been found to characterize anti-social, psycho-pathic behavior" [26]. "Brain function was evaluated in four psychiatric patients with a history of repetitive purposeless violent behaviour, using EEG, CT scan, positron emission tomography (PET). Three patients showed spiking activity in left temporal regions, and two showed CT scan abnormalities characterised by generalised cortical atrophy. The PET scans for the four cases showed evidence of blood flow and metabolic abnormalities in the left temporal lobe. 2 patients also had derangement in the frontal cortex.

The patients showing the largest defects with the PET scans were those whose CT scans were reported as normal. This work shows the utility of PET in investigating possible brain derangements that could lead to violent behaviour." "To examine if different violent offending behaviours are associated with different clinical and neuro-imaging profiles. 39 schizophrenic and schizo-affective offenders from a maximum security mental hospital -20 repetitive violent offenders (RVOs) and 19 non-repetitive violent offenders (NRVOs) - were selected for clinical and neuroimaging assessments. Both groups had positive family history of mental illness and 
violence. Age, diagnosis, duration of illness, victim profiles and use of weapons at the time of the index offence were similar. RVOs had a higher prevalence of early parental separation, juvenile conduct problem, previous convictions of crimes not involving violence, impulsive suicide attempts, delusion of their lives being threatened at the time of the index offence and electro-encephalographic (EEG) abnormalities localized to temporal lobes. NRVOs had a higher prevalence of sexual in-experience and command hallucinations to kill at the time of the index offence. Asymmetric gyral patterns at the temporo-parietal region were particularly common in RVOs and absent in NRVOs. Non-specific white matter changes in MRI and generalized cortical hypo-metabolism in PET were present in both groups.

Different structural and metabolic changes in the brain were associated with different violent offending behaviours. The complex interaction between violent behaviour, clinical features and neuroimaging findings in schizophrenia requires further studies". "Timely delivery of information is essential for proper function of the nervous system. Precise regulation of nerve conduction velocity is needed for correct exertion of motor skills, sensory integration and cognitive functions. In vertebrates, the rapid transmission of signals along the nerve fibers is made possible by the myelination of axons and the resulting saltatory conduction in between nodes of Ranvier. Myelin is a specialization of glia cells and is provided by oligo-dendrocytes in the central nervous system. Myelination not only maximizes conduction

velocity and provides a means to systematically regulate conduction times in the NS. Systematic regulation of conduction velocity along axons, and thus systematic regulation of conduction time in between neural areas, is a common occurrence in the NS. little is understood about the mechanism that underlies systematic conduction velocity regulation and conduction time synchrony. Node assembly, internode distance (node spacing) and axon diameter - all parameters determining the speed of signal propagation along axons - are controlled by myelinating glia. an interaction between glial cells and neurons has been suggested.

This review work summarizes examples of neural systems in which conduction velocity is regulated by

anatomical variations along axons. While functional implications in these systems are not always clear, recent studies in the auditory system of birds and mammals present example. of conduction velocity regulation in systems with high temporal precision and a defined biological function. these findings suggest an active process that shapes the interaction between axons and myelinating glia to control conduction velocity along axons. conduction velocity is used for the speed of signal propagation, i.e. the speed at which an action potential travel. Conduction time refers to the time it takes for a specific signal to travel from its origin to its target, i.e. neuronal cell body to axonal terminal. An example of systematic regulation of conduction velocity in mammals is found in the fibers from the inferior olive to cerebellar purkinje cells in rats. Purkinje cells in the cerebellar cortex display a high level of spiking synchronicity, supported by electrical coupling of IO cells through gap junctions. In rats, the olivocerebellar path length varies considerably between the different parts of the cerebellar cortex however their conduction times are fairly equal.

Variations in axon diameter as well as differential myelination contribute to these isochronic inputs. It should be noted that these findings are not without controversy; these results have not been observed in the cat, suggesting that the iso-chronicity of the olivo-cerebellar pathway is due to a restricted brain size in smaller animals (Aggelopoulos et al., Baker and Edgley). Another representation of intra-axonal variation of conduction velocity was illustrated in the Thalamo-cortical pathway of mice by Salami and colleagues. Neurons in the ventro-basal nucleus of the thalamus project to different areas in the cortex and their projections differ widely in length. The axon partitions in the intra-cortical region, however, cover a similar distance, due to the architecture of the cortical areas. Despite the length difference of the projections, action potentials elicited in the thalamus arrive around the same time at their target. Within cortical areas, conduction velocity slows down about 10-fold in thalamo-cortical axons, most likely the result of decreased myelination. Thus, the longer, more variable axon segments propagate action potentials quickly, while the shorter, uniform segments contribute most to overall conduction time. In the retina, variations in conduction velocity minimize conduction time differences among retinal ganglion cell axons (Stanford).

This presumably ensures spatio-temporal representation of the retinal image so that differences between conduction times to the lateral geniculate nucleus are minimized. This presumably ensures spatio-temporal representation of the retinal image so that differences between conduction times to the lateral geniculate nucleus are minimized. Another example is found in the projection from the lateral amygdala to distributed perirhinal sites. Conduction times are similar, despite the fact that the signals must travel different distances.

\section{Discussion}

In vertebrate's evolution some relevant neuronal circuit like thalamus- amygdala are present also in humans but if this circuits in reptile was an evolutive advantages in humans today can be a wrong characterisctic in actual society. accurate legal psycologic or psychiatric opinion are asked by judges but there are really objetictivated and measured results? Amygdala activation metabolism can produce different reactions in different individuals also under the same amout of stilumolous Under an evolutive approach amygdala and limbic system are "builded" to react in more rapid way versus cortex to protect the organism from Resque and dangerous stimuli. The rapid response in this way is an evolutive advantages, but the same circuits can be dangerous if activated in 
humans in actual days and society. If in voluntary crime cortex play a relevant role in non-voluntary amygdala and limbic system are involved in priority ways. In an experimental is possible to see in face money probability that $\mathrm{P}=1 / 2=0,5$ to have heads, but what happen in another situation involved humans in high stressing condition in really short time like seconds? Can we observe 0,5 of probalitity in shenario.

A (without crime) or scenario B (with crime). And what can be the response in different amygdala (anatomic- metabolic) of different subjects? How a little difference in anatomy- metabolic status can influence this kind of situation? (Figure 2) Is possible to produce a function related amygdala and limbic level of activation (metabolism, imaging or other) related an increased stressant stimulus? Basal and after high stressant stimulus and related different time of exposition (short, medium, long). Function like other phisio-pathological charactheristic like: (Figures 3-6) Why in jurisdictional contest related some non-voluntary crime is not used objective amygdala functionality profile to be added to the classic forensic psychiatric- psycologial criminology legal report? Why with forensic laboratory assay are currently detected nanoparticle or DNA molecule but not profiled amygdala full functionality.

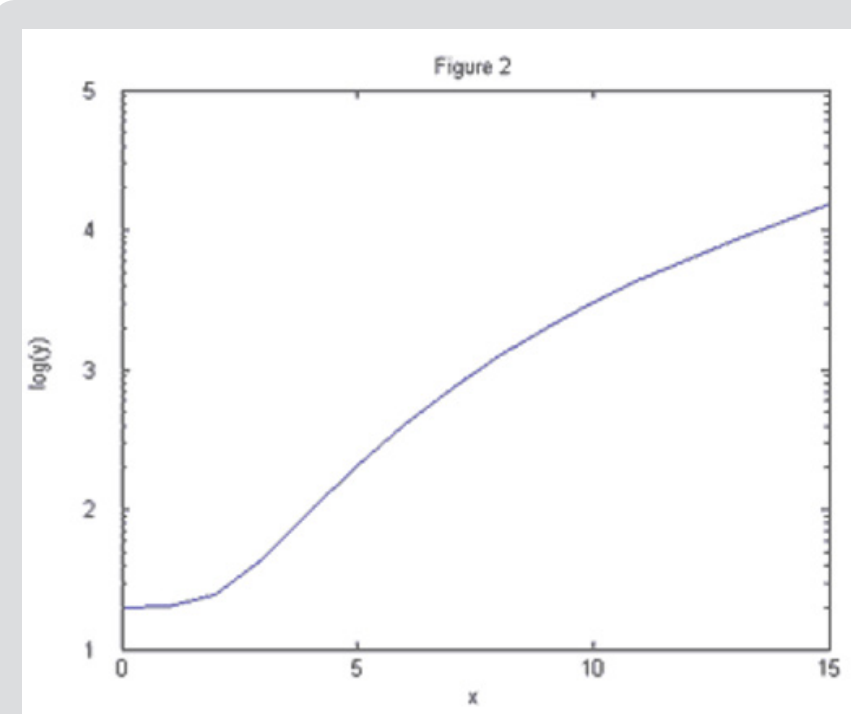

Figure 2: Mathematic logarithmic function: this approach can be used to monitoring amygdala functionality related time (in determinate stressing condition).

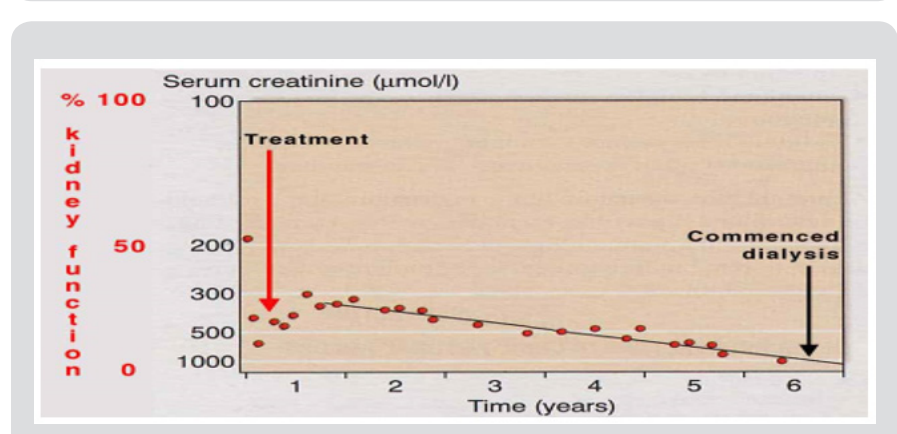

Figure 3: Renal function - times years.

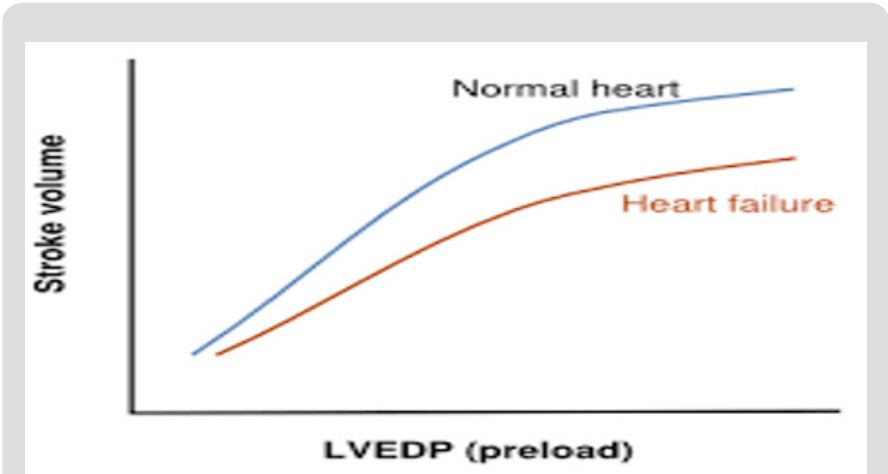

Figure 4: Heart Failure and Mathematical Function.

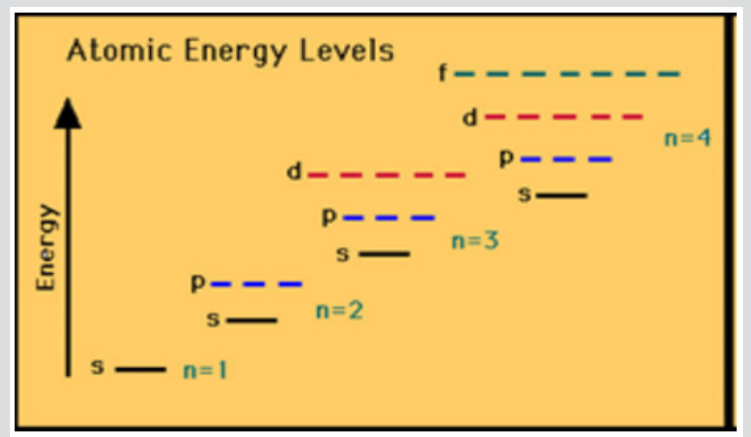

Figure 5: Quantized atomic energy (High Stressant Stimuli Can Be Considered Quantized)

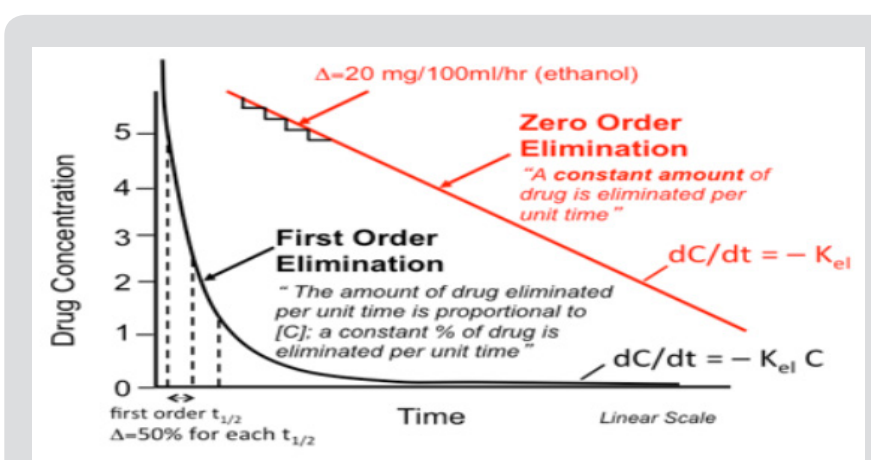

Figure 6: Kinetics Zero Order (pharmaco-toxicology) ---- example from other science to be investigated: amount and level of high stress ant stimulus and time of exposition

\section{Conclusion}

Related to the reference produced is relevant in jurisdictional setting to introduce new psycologic-psychiatric-neurology diagnostic methods ( imaging or other ) to verify level of activation of limbic system - amigdala (metabolism, substrate level , anatomy , kinetics, differential activation between cortical - amigdala and other characteristics as level of activations, imaging, nuclear medicine ) to better objectivate subject attitude to react in high stressing condition in very limited time ( emotional storm conditions). Is possible that today we can classify in example heart disease giving an numeric value in some property like heart ejection fraction , blood glucose level and after glucose charge ,renal functionality 
,body temperature, blood $\mathrm{PH}$, electromiography, urofluxometria and many other parameter and we not have possibility to objective set the amygdala basal level of activation the different humans in really high stressing condition time related ? (imaging like f MRI, PET, SPECT, anatomy, lesions, biochemistry, substrate, neurotrasmitters , blood flux rate, time of response, basal and after high stressing condition in restricted time and other useful parameter ), To measure related different level of stressanting condition ( amount and kinetics , saturation of the system ) in restricted time in different humans make possible to verify phisio-patologial responce Or abnormality in reaction.

A global index of amygdala functionality - activation level can be: AMYGDALA func- activ -level INDEX $=\log$ function of A (anatomic- imaging - abnormality - local pathology ) x B( physiology abnormality : metabolic substrate, methabolism ) x C ( neuronal circuit conductivity- velocity of neuronal circuits - difference from average). In our opinion in jurisdictional settings in some non-voluntary crime to verify the really objective amygdala functionality profile to be added to the classic forensic psychiatric- psycologial criminology legal report is relevant and crucial to add also amygdala and limbic system functionality instrumental - imaging - biochemical assay. Not a prove but an objective data to be considered in contestualizyng the frame of the facts. In example In a car crash a diabetic driver whit blood glucose level at $450 \mathrm{mg} / \mathrm{dl}$ how can control his car? and in this situation what will be the profile of responsibility? In 1700-1800 periods physician not used to test patients' blood glucose level but this safelife parameter is currently tested in every emergency patient without any problems. The same cardiac artimia is normally classified related the single cell membrane channel abnormality (K, NA, CA). Why amygdala in not tested like other organ and system? In example cardiac, renal, respiratory function? Concepts from other science like biochemistry, toxicology, kinetics, imaging and many other contribute to better clarify some organic situation that can be co- responsible of serious concequences.

\section{References}

1. Mauro Luisetto, Khan FA, Cabianca L, Mokbul MI, Rafa AY, et al. (2016) Amygdala pharmacology andcrime behavior, dysfunctions to be considered as a disease. Int Arch BioMed Clin Res 2(2).

2. (2017) $7^{\text {th }}$ Global Experts Meeting on Neuropharmacology. Theme Neuropharmacology: Major Challenges and Breakthroughs Milan, Italy.

3. Mindset Kinetics Under Toxicological Aspect Psychology and Behavioral Science International Journal ISSN 2474-7688 8(2).

4. Luisetto M (2017) Brain and Transmission Signal Modulation. Theranostics Brain Disord 1(1): TBD.MS.ID.555553.

5. Luisetto, Naseer Almukhtar, Ghulam Rasool Mashori, Ahmed Yesvi Rafa, Farhan Ahmad Khan, et al. Endogenous archeological sciences: Physiology, Neuroscience, Biochemistry, Immunology, Pharmacology, Oncology and Genetics as instrument for A New Field of Investigation? Modern Global Aspects for A New Discipline 1(3).

6. For a new field of investigation? Modern global aspects for a new discipline Journal of Neuroscience and Neurological Disorders.

7. Luisetto M, Mashori GR, Nili Ahmadabadi B, Khan FA, Khan KR (2018) Mindset kinetics and some depression status: A new quantitative model under biochemical - toxicology approach? Insights Depress Anxiety 2: 029-039.

8. J Law Biosci, Uri Maoz, Gideon Yaffe (2016) What does recent neuroscience tell us about criminal responsibility? J Law Biosci 3(1): 120-139.

9. Sergio Canavero (2014) Criminal Minds: Neuromodulation of the Psychopathic Brain. Front Hum Neurosci 8: 124.

10. Rebecca Roache (2014) Can brain scans prove criminals unaccountable? AJOB Neurosci 5(2): 35-37.

11. Dean Mobbs, Hakwan C Lau, Owen D Jones, Christopher D Frith (2007) Law, Responsibility, and the Brain. PLoS Biol 5(4): e103.

12. Robert K Naumann, Janie M Ondracek, Samuel Reiter, Mark Shein Idelson, Maria Antonietta Tosches, et al. (2015) The reptilian brain. Curr Biol. 25(8): R317-R321.

13. Hoerst M, Weber Fahr W, Tunc Skarka N, Ruf M, Bohus M, et al. (2010) Metabolic alterations in the amygdala in borderline personality disorder: a proton magnetic resonance spectroscopy study. Biol Psychiatry 67(5): 399-405.

14. Sakai Y, Kumano H, Nishikawa M, Sakano Y, Kaiya H, et al. (2005) Cerebral glucose metabolism associated with a fear network in panic disorder Neuro report 16(9): 927-931.

15. Cunha Bang, Patrick M Fisher, Liv Vadskjær Hjordt, Erik Perfalk, Anine Persson Skibsted, et al. (2017) Violent offenders respond to provocations with high amygdala and striatal reactivity. Sofi daSoc Cogn Affect Neurosci 12(5): 802-810.

16. Larry J Siever (2008) Neurobiology of Aggression and Violence. Am J Psychiatry 165(4): 429-442.

17. Lara C Foland Ross, Lori L Altshuler, Susan Y Bookheimer, Matthew D Lieberman, Jennifer Townsend, et al. (2010) Amygdala reactivity in healthy adults is correlated with prefrontal cortical thickness. J Neurosci 30(49): 16673-16678.

18. Roee Admon, Gad Lubin, Orit Stern, Keren Rosenberg, Lee Sela, et al. (2009) Human vulnerability to stress depends on amygdala's predisposition and hippocampal plasticity PNAS 106(33): 14120-14125.

19. Altshuler L, Bookheimer S, Proenza MA, Townsend J, Sabb F, et al. (2005) Increased amygdala activation during mania: a functional magnetic resonance imaging study. Am J Psychiatry 162(6): 1211-1213.

20. Sofi da Cunha Bang, Patrick M Fisher, Liv Vadskjær Hjordt, Erik Perfalk, Anine Persson Skibsted, et al. (2017) Violent offenders respond to provocations with high amygdala and striatal reactivity. Soc Cogn Affect Neurosci 12(5): 802-810.

21. M Brower, B Price (2001) Neuropsychiatry of frontal lobe dysfunction in violent and criminal behaviour: a critical review. J Neurol Neurosurg Psychiatry 71(6): 720-726.

22. Hirono N, Mega MS, Dinov ID, Mishkin F, Cummings JL (2000) Left frontotemporal hypoperfusion is associated with aggression in patients with dementia. Arch Neurol 57(6): 861-866.

23. Raine A, Lencz T, Bihrle S, La Casse L, Colletti P (2000) Reduced prefrontal gray matter volume and reduced autonomic activity in antisocial personality disorder. Arch Gen Psychiatry 57(2): 119-127.

24. Volkow ND, Tancredi L (1987) Neural substrates of violent behavior. A preliminary study with positron emission tomography. Br J Psychiatry 151: 668-673

25. (1997) Repetitive and Non-Repetitive Violent Offending Behaviour in Male Patients in a Maximum-Security Mental Hospital - Clinical and Neuroimaging Findings Michael Wong, MRC Psych, Peter Fenwick, FRC Psych, George Fenton et al Medicine, Science and the Law 1997.

26. Armin H Seidl (2015) Regulation of Conduction Time along Axons. Neuroscience pp. 126-134. 
ISSN: 2574-1241

DOI: 10.26717/BJSTR.2019.16.002810

Mauro Luisetto. Biomed J Sci \& Tech Res

(C) This work is licensed under Creative

Submission Link: https://biomedres.us/submit-manuscript.php

\begin{tabular}{ll} 
BIOMEDICAL & \multicolumn{1}{c}{ Assets of Publishing with us } \\
RESEARCHES & - Global archiving of articles \\
\hline ISSN:2574-1241 & - Immediate, unrestricted online access \\
\end{tabular}

